\title{
Annular pancreas: endoscopic and pancreatographic findings from a tertiary referral ERCP center
}

Mark A. Gromski, Glen A. Lehman, Nicholas J. Zyromski, James L. Watkins, Ihab I. El Hajj, Damien Tan, Lee McHenry, Jeffrey J. Easler, Temel Tirkes, Stuart Sherman, Evan L. Fogel

\section{ABSTRACT}

Background and Aims: Annular pancreas is a congenital anomaly where pancreatic tissue encircles the duodenum. Current knowledge of endoscopic findings of annular pancreas is limited to small case series. The aim of this study is to describe the endoscopic and pancreatographic findings of patients with annular pancreas at a large tertiary care ERCP center.

Methods: This is a retrospective observational study. Our IRB approved, prospectively collected ERCP database was queried for cases of annular pancreas. The electronic medical record was searched for patient and procedure-related data.

Results: From 1/1/94 - 12/31/16, 46 patients with annular pancreas underwent ERCP at our institution. Index ERCP was technically successful in 42 patients (91.3\%) and technical success was achieved in all 46 patients (100\%) after two attempts, when required. A duodenal narrowing or ring was found in the majority of patients ( $n=39,84.8 \%)$, yet only $2(4.3 \%)$ had retained gastric contents. Pancreas divisum was found in 21 patients (45.7\%), 18 of which were complete divisum. Pancreatobiliary neoplasia was the indication of ERCP in 7 patients (15.2\%). Pancreatographic findings consistent with chronic pancreatitis were noted in 15 patients $(32.6 \%)$ at the index ERCP.

Conclusion: This is the largest series describing the endoscopic and pancreatographic findings of patients with annular pancreas. We found that $45.7 \%$ of patients had concurrent pancreas divisum. Endoscopic therapy was successful in the vast majority of patients at our institution after one ERCP, and in all patients after a second ERCP. Nearly one-third of patients had findings consistent with chronic pancreatitis at the time of index ERCP. It is unclear whether this may be a feature of the natural history of annular pancreas.

KEYWORDS: annular pancreas, ERCP, pancreas divisum 


\section{Introduction:}

Annular pancreas is a rare congenital anomaly, often occurring in children who present with duodenal obstruction. Annular pancreas, however, has been diagnosed with nearly equal frequency in pediatric and adult patients. ${ }^{1}$ It is a pathological entity where pancreatic tissue encircles the duodenum either completely or nearly completely (Figures 1, 2 and 3). The embryological defect in development has been postulated to be from an abnormal fusion of the ventral pancreatic bud tip to the duodenum. This results in abnormal rotation of the ventral bud around the duodenum during early embryological development, and ultimately the ringed or semi-ringed pancreatic tissue around the second portion of the duodenum that is known as annular pancreas. ${ }^{1}$ Concurrent pancreas divisum has been observed more frequently in patients with annular pancreas compared to the normal population, with a reported concurrence rate in adults of $29-36 \%$ in previous series and literature reviews. ${ }^{1,2}$

The incidence of annular pancreas has been difficult to determine precisely, but based on autopsy and diagnostic population studies, the incidence is estimated to be between 1 in 1000 and 1 in 6,000 people. ${ }^{1,3-5}$ The endoscopic findings of annular pancreas have not been clearly defined. In a large series, Zyromski et al. compared the clinical findings of pediatric to adult patients with annular pancreas, but significant endoscopic details were not included in this report. ${ }^{1}$ Furthermore, a study by Sandrasegaran et al details the radiographic findings in a large series of adult patients with annular pancreas. ${ }^{6}$ There are, however, no large series describing the endoscopic and pancreatographic findings of patients with annular pancreas. The endoscopic 
literature to date has been limited to case reports. ${ }^{2,7-18}$ The aim of this study is to describe the endoscopic and pancreatographic findings of a large series of patients with annular pancreas at a large tertiary care pancreatobiliary endoscopy center.

\section{Methods:}

This is a retrospective, observational study describing the endoscopic and pancreatographic findings of patients with annular pancreas undergoing ERCP. The study was approved by the institutional review board (IRB) at Indiana University Hospital. The single inclusion criterion for study entry was presence of annular pancreas, identified either surgically, radiographically or endoscopically, in a patient who underwent an ERCP at our institution from January 1994 to December 2016. The patients were identified from our IRB-approved, prospectively maintained ERCP database and the electronic procedure reporting system (ProVation Medical, Minneapolis, MN). The databases were searched for findings of annular pancreas. A retrospective review of clinical data was performed, including a query of the electronic medical record and the electronic procedure reporting system. The following clinical data and demographics were collected for the study patients: age at time of index ERCP, gender, race, indication for ERCP, clinical presentation/symptoms, history or presence of pancreatobiliary malignancy, endoscopic findings (including presence of retained gastric contents, location of minor papilla, presence of annular ring), pancreatographic findings (including presence of pancreas divisum [complete or incomplete], visualization of annular ring, presence of pancreatographic findings of chronic pancreatitis at time of index ERCP), and technical success. For this study, a diagnosis of annular 
pancreas was made at the time of ERCP if there was pancreatographic evidence of a pancreatic duct encircling the area of the duodenum. This was supported by the presence of a narrowing or ring on endoscopic evaluation (although not necessary for diagnosis). A diagnosis of annular pancreas from surgery or a radiographic study was determined if the final radiographic (CT or MRI) report or operative report by the attending physician noted findings consistent with annular pancreas (generally noted to be pancreatic tissue encircling the duodenum). Technical success at ERCP was defined as cannulation of the desired duct and completion of the desired therapeutic maneuver (when required) planned by the endoscopist. Descriptive statistics were used to present the data.

\section{Results:}

From 01/01/94 - 12/31/16, 46 patients with annular pancreas underwent ERCP at our institution. Patient characteristics are described in Table 1. The majority of patients were female $(n=33,71.7 \%)$ and Caucasian $(n=40,87.0 \%)$. The mean age at index ERCP was $46.3 \pm$ 17.2 years (range $4-76$ years). Two patients were less than 18 years old. One of these patients was 7 years old and had a prior duodenojejunostomy. Five total patients (10.9\%) had prior foregut surgery to address annular pancreas, including three patients with prior duodenojejunostomy and two patients with prior duodenoduodenostomy. The indications for ERCP are also summarized in Table 1. Many patients had more than one indication (e.g., idiopathic recurrent acute pancreatitis [IRAP] and suspected sphincter of Oddi dysfunction). Of 
the 46 patients in this study, 40 patients had an indication with a potential pancreatic pathology.

All patients included in the study had an ERCP performed and diagnosis of annular pancreas made (either prior to ERCP or at ERCP). The initial diagnosis of annular pancreas was made at ERCP in 31 patients (67.4\%). The diagnosis of annular pancreas had previously been made (prior to $\mathrm{ERCP})$ by $\mathrm{CT}$ or MRI/MRCP in 8 patients (17.4\%). Five patients had previously been diagnosed with annular pancreas from a previous surgery (10.9\%). The method of index diagnosis could not be determined in two patients (4.3\%) due to incomplete outside medical records preceding index ERCP.

Pancreatographic Findings and Technical Success

A pancreatogram was performed in all 46 patients. A summary of the pancreatographic findings are detailed in Table 2. Technical success was achieved on index ERCP in 42 patients (91.3\%) and technical success was achieved in 46 patients (100\%) after two attempts, when required. The annular branch was visualized on pancreatogram in 34 patients (73.9\%), arising from the ventral pancreatic duct in 30 of 34 patients (88.2\%, Figure 5). Pancreas divisum was found in 21 patients (45.7\%), 18 of which were complete divisum. Three of the four patients who did not realize technical success on the index ERCP had pancreas divisum and intended therapy at the minor papilla. Technical success was achieved at index ERCP in 18 of 21 patients with concurrent pancreas divisum (85.7\%), while technical success was achieved in 24 of the 
remaining 25 patients without concurrent pancreas divisum (96.0\%). Findings consistent with chronic pancreatitis (based on the Cambridge classification) ${ }^{19}$ were noted on the index pancreatogram in 15 patients (32.6\%). Pancreatographic findings consistent with chronic pancreatitis were found in $38 \%$ of annular pancreas patients with pancreas divisum (8/21) and $28 \%$ of annular pancreas patients without pancreas divisum (7/25). Of the 15 patients with pancreatographic changes of chronic pancreatitis, chronic pancreatitis changes were found in the main duct in $12(80 \%)$ of patients, in the annular branch in $3(20 \%)$ of patients. Furthermore, the changes were specified to be located in $6(40 \%)$ patients in the ventral duct and 5 (33.3\%) patients in the dorsal duct. An anomalous pancreatobiliary junction (APBJ) was identified in 1 patient (2.2\%).

Two of the 46 patients (4.3\%) had a complication related to the procedure: one patient had mild post-procedure pancreatitis and one patient had post-procedure GI bleeding (postsphincterotomy).

\section{Endoscopic Findings}

Of the 41 patients without prior foregut surgery, a duodenal narrowing or ring (Figure 3) was found in the majority of patients $(n=36,87.8 \%)$, yet only $2(4.9 \%)$ had retained gastric contents. Of the 21 patients with pancreas divisum, the location of the minor papilla was reported in 16 patients. In six patients (37.5\%), the minor papilla was located proximal to the annular narrowing. In five patients (31.3\%), the minor papilla was located on the annular narrowing 
itself. One patient (6.3\%) was noted to have the minor papilla distal to the annular narrowing.

Two patients (12.5\%) were noted to have the minor papilla in the "usual" or "normal" position. Two patients (12.5\%) were noted to have the minor papilla at the transition of the duodenal bulb and second portion of the duodenum, without mention of a relation to the annular ring. Representative images of the location of the minor papilla with relation to the annular narrowing in patients with concurrent annular pancreas and pancreas divisum are shown in Figure 4.

\section{Associated Findings}

Pancreas sphincter manometry was performed in 9 patients (19.6\%, all but one of these patients did not have pancreas divisum). Of these patients, six (66.7\%) had abnormal pancreatic sphincter manometry, confirming a diagnosis of pancreatic SOD. Pancreatobiliary neoplasia was identified in 7 patients (15.2\%; pancreatic adenocarcinoma $=3$, ampullary neoplasia $=2$, gallbladder cancer $=1$, main-duct intraductal papillary mucinous neoplasm $=1$ ) . Moreover, four other patients had non-pancreatobiliary malignancy $(8.7 \%$; colon cancer $=1$, lymphoma $=1$, breast cancer $=1$, prostate cancer $=1$ ). Therefore, $23.9 \%$ of patients had either pancreatobiliary neoplasia or other cancer. Of the 7 patients with pancreatobiliary neoplasia, three patients had a concurrent second malignancy (one patient with gallbladder cancer, ampullary adenoma and prostate cancer, one patient with pancreatic adenocarcinoma and leukemia, and one patient with pancreatic adenocarcinoma and prostate cancer). 


\section{Discussion:}

This study describes the endoscopic and pancreatographic findings of 46 patients with annular pancreas who underwent ERCP over a period of 23 years at a single center in the United States. Prior reports of endoscopic and pancreatographic findings of patients with annular pancreas have been limited to case reports or reviews of the literature of previously published cases. Given the rarity of this congenital condition, this is the largest series to date describing the endoscopic and pancreatographic findings of patients with annular pancreas.

\section{$\underline{\text { Procedural considerations }}$}

At our tertiary referral ERCP center, less than $2 \%$ of procedures are performed in children or adolescents (age < 18). While children with annular pancreas may undergo surgery for upper GI obstructive symptoms, adults appear to present to medical attention with pancreatobiliary pathology. Interestingly, despite the majority of patients (87.8\%) in this series having a duodenal ring noted on ERCP, less than $5 \%$ of patients had retained gastric contents noted during endoscopic examination, and none severe enough to require the ERCP to be aborted. This number is surprisingly low, given the potential for other factors which might have contributed to retained gastric contents (e.g., opioid use, diabetic gastroparesis, etc). It may be possible that there are two phenotypes of patients with annular pancreas - the first being a phenotype where the annular ring constricts the duodenum causing gastric outlet obstruction, and the second being a phenotype where the annular ring is relatively loose or incomplete. The former most likely presents in childhood and is managed surgically. 
Based on the indications for ERCP, it is not clear that the annular pancreas in itself is the driving factor behind the need for ERCP in all patients in this study. Indeed, it may be an innocent bystander, as in the case of a patient with biliary SOD. It is difficult to ascribe any meaningful role of annular pancreas in the patient with right upper quadrant abdominal pain and elevated liver function tests, and it almost certainly represents an incidental finding in this situation. The study also demonstrated that, despite having features that traditionally make diagnostic and therapeutic maneuvers at ERCP more difficult, including duodenal deformity and frequent need for minor papilla access, ERCPs were able to be performed with complete success in the majority of cases $(91.3 \%)$ on the first attempt. Technical success was achieved in all patients after a second ERCP in those who did not have technical success after a first procedure. Given the large proportion of patients in this study with pancreas divisum (45.7\%), 11 patients (23.9\%) underwent minor papilla cannulation for intended therapy. Over half of the patients with pancreas divisum had the minor papilla located either proximal to or on the annular ring (Figure 6), which likely further increased the technical difficulty of procedures in this cohort.

\section{Annular pancreas and pancreas divisum}

In a review of 14 cases of annular pancreas published in the English literature in and before 1985, Lehman and O'Connor found that 5 of these cases (36\%) had coexistent annular pancreas and pancreas divisum. ${ }^{2}$ This suggested that pancreas divisum occurs more often in the presence of annular pancreas than it does in the general population (5-10\%). ${ }^{20,21}$ In a review of CT, MRI, and ERCP findings of annular pancreas in adults, Sandrasegaran et al. reported a similar rate of 
pancreas divisum (37\%). ${ }^{6}$ In a series by Zyromski et al., 16 adults (29\%) were diagnosed with pancreas divisum, which was significantly more often than children, in whom the condition was not observed. ${ }^{1}$ This increased coexistent defect is not surprising in as much as the rotational error resulting in annular pancreas changes the orientation of the dorsal and ventral pancreatic ducts that normally fuse. ${ }^{22}$ In the current study, pancreas divisum was found in 21 patients (45.7\%). This is somewhat higher than the previously reported rate of coexisting conditions, although earlier reports were limited by small patient numbers. ${ }^{2}$ This may indicate that there is a larger proportion of adult patients with coexistent annular pancreas and pancreas divisum among those referred to ERCP for evaluation of pancreatobiliary pathology.

\section{Annular pancreas and chronic pancreatitis}

Nearly one-third of patients (32.6\%) had findings on ERP suggestive of chronic pancreatitis at the time of index ERCP in this series. It is unclear whether this may be a feature of the natural history of annular pancreas. Given the presenting phenotype of the patient (symptoms, risk factors, etc.) cannot be precisely determined in this retrospective study over multiple decades, it is not possible to ascribe the pancreatographic findings alone to a diagnosis of chronic pancreatitis. Obstructive pathology may play a role in the etiology of pancreatographic findings suggestive of chronic pancreatitis, as 26 patients (56.5\%) in this study were found to have either pancreatic SOD or pancreas divisum at the time of ERCP. Indeed, of the 15 patients found to have findings of chronic pancreatitis at index ERCP, 11 (73.3\%) had either concurrent abnormal manometry or pancreas divisum. There was a higher rate of pancreatographic findings of chronic pancreatitis in those who had concurrent pancreas divisum (38\%) compared 
to those without pancreas divisum (28\%). Pancreas divisum is a known risk factor for chronic pancreatitis.

\section{Annular pancreas and malignancy}

In a review of 14 cases of annular pancreas and concurrent pancreatobiliary malignancy published in the English literature in and before 2007, the most commonly identified cancer was ampullary carcinoma (6 cases), followed by pancreatic carcinoma (5 cases), cholangiocarcinoma ( 2 cases), and insulinoma ( 1 case). ${ }^{27}$ This has previously been attributed to chronic inflammatory changes in this area associated with pancreatitis. In their series of 103 patients (48 children, 55 adults), Zyromski et al. detected neoplasia of some type in 18 (32\%) adults and none in children $(p<0.01) .{ }^{1}$ In our current series, $23.9 \%$ of patients had either pancreatobiliary neoplasia or other cancer. These rates of pancreatobiliary neoplasia and malignancy are higher than expected in the general population. For many of the patients presenting for ERCP in this study, pancreatobiliary malignancy was the cause of the indication of the ERCP procedure (e.g., jaundice or imaging abnormality), thus there is likely a selection bias in this population of patients with concurrent annular pancreas and malignancy. A causal relationship with annular pancreas cannot be determined from this study.

\section{$\underline{\text { Annular pancreas and } A P B J}$}

Twelve cases of concurrent annular pancreas and APBJ have been reported in the English literature, of which 11 were pediatric cases. ${ }^{28}$ Data from these reports show that most of these patients underwent duodenoduodenostomy and flow-diversion surgery simultaneously or 
metachronously. In our experience, APBJ was noted in one (2.2\%) patient. This finding may be explained by the fact that our patient population was adult-predominant.

\section{Limitations:}

We recognize several limitations to our study, including selection bias. We used our internal ERCP database to identify subjects eligible for the study. In other words, we used ERCP as the reference standard for determining the presence of annular pancreas. This was the methodology selected, as the goal of this study was to describe endoscopic findings and pancreatographic features of patients that undergo ERCP for annular pancreas. Patients found to have annular pancreas by other imaging techniques who did not subsequently undergo ERCP were not evaluated. Furthermore, the procedures in this study were performed by endoscopists with expertise in pancreatic endotherapy and in a very busy pancreatobiliary referral center (tertiary center referral bias), thus the outcomes may not be generalizable to facilities with less experience. The majority of patients were Caucasian and female - this may be explained by the large number of patients undergoing ERCP for suspicion of SOD, which predominates in Caucasian females.

In summary, this study describes the endoscopic and pancreatographic findings of 46 patients with annular pancreas who underwent ERCP at a single center over a period of 23 years. This is a finding found rarely at the time of ERCP. Despite the potential for altered duodenal and pancreatic anatomy, endoscopic success rates remain high by experienced ERCP endoscopists. 


\section{References}

1. Zyromski NJ, Sandoval JA, Pitt HA, et al. Annular pancreas: dramatic differences between children and adults. Journal of the American College of Surgeons 2008;206:1019-25; discussion 1025-7.

2. Lehman GA, O'Connor KW. Coexistence of annular pancreas and pancreas divisum--ERCP diagnosis. Gastrointestinal endoscopy 1985;31:25-8.

3. Theodorides T. [Annular Pancreas]. Journal de chirurgie 1964;87:445-62.

4. Chevillotte G, Sahel J, Raillat A, et al. Annular pancreas. Report of one case associated with acute pancreatitis and diagnosed by endoscopic retrograde pancreatography. Digestive diseases and sciences 1984;29:75-7.

5. Glazer GM, Margulis AR. Annular pancreas: etiology and diagnosis using endoscopic retrograde cholangiopancreatography. Radiology 1979;133:303-6.

6. Sandrasegaran K, Patel A, Fogel EL, et al. Annular pancreas in adults. AJR. American journal of roentgenology 2009;193:455-60.

7. Urayama S, Kozarek R, Ball T, et al. Presentation and treatment of annular pancreas in an adult population. The American journal of gastroenterology 1995;90:995-9.

8. Ladd AP, Madura JA. Congenital duodenal anomalies in the adult. Archives of surgery 2001;136:576-84.

9. Clifford KM. Annular pancreas diagnosed by endoscopic retrograde-choledochopancreatography (ERCP). Br J Radiol 1980;53:593-5.

10. Adamo M, Bonventre $S$, Tavormina A, et al. Severe obstructive jaundice in a 90-year-old man caused by an annular pancreas. Report of a case. Minerva Chir 2003;58:395-7.

11. Benger JR, Thompson MH. Annular pancreas and obstructive jaundice. The American journal of gastroenterology 1997;92:713-4.

12. Dharmsathaphorn K, Burrell M, Dobbins J. Diagnosis of annular pancreas with endoscopic retrograde cholangiopancreatography. Gastroenterology 1979;77:1109-14.

13. England RE, Newcomer MK, Leung JW, et al. Case report: annular pancreas divisum--a report of two cases and review of the literature. Br J Radiol 1995;68:324-8.

14. Itoh Y, Hada T, Terano A, et al. Pancreatitis in the annulus of annular pancreas demonstrated by the combined use of computed tomography and endoscopic retrograde cholangiopancreatography. The American journal of gastroenterology 1989;84:961-4.

15. Perez de Ayala V, Cebria L, Castellanos D, et al. Association of annular pancreas and pancreas divisum. Endoscopy 1991;23:310-1.

16. Rassadi R, Jolley TM, Saad AJ, et al. A rare form of annular pancreas: annular duct originating from the duct of Santorini. Gastrointestinal endoscopy 2008;67:544-5.

17. Yogi Y, Shibue T, Hashimoto S. Annular pancreas detected in adults, diagnosed by endoscopic retrograde cholangiopancreatography: report of four cases. Gastroenterol Jpn 1987;22:92-9.

18. Dowsett JF, Rode J, Russell RC. Annular pancreas: a clinical, endoscopic, and immunohistochemical study. Gut 1989;30:130-5.

19. Sarner M, Cotton PB. Classification of pancreatitis. Gut 1984;25:756-9.

20. MacCarty RL, Stephens DH, Brown AL, Jr., et al. Retrograde pancreatography in autopsy specimens. The American journal of roentgenology, radium therapy, and nuclear medicine 1975;123:359-66.

21. Stimec B, Bulajic $M$, Tatic $S$, et al. Unusual variants of the tributaries of the main pancreatic duct revealed by postmortem and endoscopic pancreatography. Annals of anatomy = Anatomischer Anzeiger : official organ of the Anatomische Gesellschaft 1996;178:169-71. 
22. White JJ, Roberts ZN, Gest TR, et al. Pancreas divisum: a common developmental variant that deserves attention in preclinical medical education. Clinical anatomy 2014;27:1038-45.

23. Duell EJ, Lucenteforte $\mathrm{E}$, Olson $\mathrm{SH}$, et al. Pancreatitis and pancreatic cancer risk: a pooled analysis in the International Pancreatic Cancer Case-Control Consortium (PanC4). Annals of oncology : official journal of the European Society for Medical Oncology 2012;23:2964-70.

24. Ekbom A, McLaughlin JK, Nyren O. Pancreatitis and the risk of pancreatic cancer. The New England journal of medicine 1993;329:1502-3.

25. Karlson BM, Ekbom A, Josefsson S, et al. The risk of pancreatic cancer following pancreatitis: an association due to confounding? Gastroenterology 1997;113:587-92.

26. Lowenfels AB, Maisonneuve P, Cavallini G, et al. Pancreatitis and the risk of pancreatic cancer. International Pancreatitis Study Group. The New England journal of medicine 1993;328:1433-7.

27. Foo FJ, Gill U, Verbeke CS, et al. Ampullary carcinoma associated with an annular pancreas. JOP : Journal of the pancreas 2007;8:50-4.

28. Cheng L, Tian F, Zhao T, et al. Annular pancreas concurrent with pancreaticobiliary maljunction presented with symptoms until adult age: case report with comparative data on pediatric cases. BMC gastroenterology 2013;13:153. 
Table 1: Characteristics of Patients with Annular Pancreas Undergoing ERCP

\begin{tabular}{|c|c|c|}
\hline Characteristic & $\mathbf{N}$ & $\%$ \\
\hline \multicolumn{3}{|l|}{ Sex } \\
\hline Female & 33 & 71.7 \\
\hline Male & 13 & 28.3 \\
\hline \multicolumn{3}{|l|}{ Ethnicity } \\
\hline White & 40 & 87.0 \\
\hline African-American & 3 & 6.5 \\
\hline Hispanic & 2 & 4.3 \\
\hline Other & 1 & 2.2 \\
\hline Age (mean +/- SD) & $46.3 \pm 17.2$ & \\
\hline Prior foregut surgery & 5 & 10.9 \\
\hline \multicolumn{3}{|l|}{ Indication for ERCP* } \\
\hline Pain / Suspicion of SOD & 20 & 43.5 \\
\hline $\begin{array}{l}\text { Idiopathic Acute or } \\
\text { Recurrent Acute Pancreatit }\end{array}$ & 17 & 37.0 \\
\hline Abnormal Imaging** & 12 & 26.1 \\
\hline Elevated liver tests & 10 & 21.7 \\
\hline Chronic pancreatitis & 7 & 15.2 \\
\hline Ampullary mass & 2 & 4.3 \\
\hline
\end{tabular}

SD, standard deviation; SOD, Sphincter of Oddi Dysfunction 
*Note: many patients have more than one indication for the ERCP, thus the \% adds up to $>100 \%$.

**Abnormal imaging consists of the following: annular pancreas $(n=5)$, dilated common bile duct $(n=3)$, pancreas cyst/pseudocyst $(n=2)$, pancreas stricture / pancreas duct dilation $(n=1)$, choledocholithiasis $(n=1)$.

Table 2: Pancreatographic Findings at ERCP for Patients with Annular Pancreas

\begin{tabular}{|c|c|c|}
\hline \multicolumn{1}{|c|}{ Finding } & N & \% \\
\hline Annular pancreas branch identified & 34 & 73.9 \\
\hline Annular branch arising & 30 & $88.2^{*}$ \\
from ventral duct or ventral & & \\
\hline Panp in divisum & 21 & 45.7 \\
\hline Pancreatographic findings & 15 & 32.6 \\
consistent with chronic & & 2.2 \\
\hline pancreatitis^ & & \\
\hline APBJ & & \\
\hline
\end{tabular}

*percentage based off of the 34 patients in which annular pancreas was identified.

^based upon Cambridge classification of pancreatographic changes

APBJ - anomalous pancreatobiliary junction 


\section{FIGURE LEGEND}

Figure 1: T1 weighted fat-saturated post-contrast MR image demonstrating complete encircling of duodenum with pancreatic tissue (arrow) in a patient with annular pancreas.

Figure 2: Coronal thick-slab MRCP image demonstrating ventral annular ring (arrows).

Figure 3: Double-contrast fluoroscopic image of an upper Gl examination showing circumferential narrowing (arrows) of the second portion of the duodenum in a patient with annular pancreas.

Figure 4. A.) Endoscopic image of circumferential narrowing of duodenum in a patient with annular pancreas, with minor papilla noted (yellow arrow) on rim of annulus. B.) Minor papilla sphincterotomy in a patient with annular pancreas and pancreas divisum.

Figure 5. A.) Ventral pancreatogram demonstrating annular duct (yellow arrows), with otherwise normal ductal anatomy (i.e. no pancreas divisum). B and C.) Ventral pancreatogram demonstrating annular duct (yellow arrows), with pancreatic divisum anatomy (no communication with main pancreatic duct). D.) Ventral pancreatogram demonstrating incomplete annular duct (yellow arrows) and findings of main duct suspicious for chronic pancreatitis including dilated main duct and prominent and irregular side branches.

Figure 6: Fluoroscopic image demonstrating paucity of air at the level of annular pancreas ring (arrow), with minor papilla cannulation and pancreatogram. The minor papilla is located at the rim of the annular pancreas (demonstrated by cannulation at level of paucity of air), which can increase difficulty. 
